\title{
Chronische Urtikaria als Folge einer SIT?
}

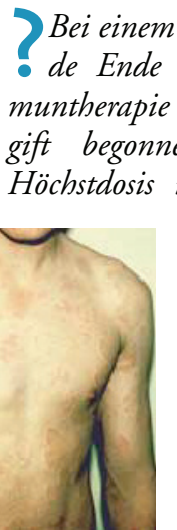

Bei einem 40-jährigen Patienten wurde Ende 1999 eine spezifische Immuntherapie mit lyophilisiertem Wespengift begonnen. Nach Erreichen der Höchstdosis im Januar 2000 ereignete sich eine anaphylaktoide Reaktion, worauf die Behandlung abgebrochen wurde.

Seit dieser Zeit kommt es bei dem Patienten zu einer generalisierten Urtikaria, die mit $2 \times 10 \mathrm{mg}$ Cetirizin nur unzureichend unterdrückt werden kann. Durch systemische Kortikosteroide lässt sich die Urtikaria erfolgreich unterdrücken. Eine Typ-I-Allergie gegen die häufigsten inhalativen und nutritiven Allergene lässt sich nicht nachweisen.

Besteht ein Zusammenhang mit der vorausgegangenen Wespengift-Hyposensibilisierung? Sind ähnliche Kasuistiken in der Literatur bekannt?

Dr. R. Golsong, Oberhausen
E in möglicher Zusammenhang mit - der spezifischen Hyposensibilisierung wird nicht vollständig auszuschließen sein. Es bestehen in der Literatur einige Hinweise auf den $\mathrm{Zu}$ sammenhang zwischen InsektengiftHyposensibilisierungen und insbesondere der Kälteurtikaria. [s. a. Hertl M, Merk HF. Occurrence of cold urticaria in insect venom allergic individuals. Allergo J 1994; 3: 379-81]

\section{Prof. Dr. Hans F. Merk}

Klinik für Dermatologie und Allergologie, Universitätsklinikum der RWTH,

Pauwelsstr. 30, D-52057 Aachen

rotz einzelner Fallberichte einer Kälteurtikaria im Zusammenhang mit einer Wespengift-Hyposensibilisierung gibt es keinerlei Hinweise auf die Auslösung einer chronischen generalisierten Urtikaria durch diese Behandlung. Grundsätzlich ist dies auch nicht zu erwarten, da es bei der Hyposensibilisie- rung nicht zu einer längerfristigen Antigen-Präsenz kommt. Diagnostisch und therapeutisch sollte daher wie bei jeder anderen chronischen Urtikaria vorgegangen werden.

Hierbei ist eine KortikosteroidDauertherapie nach Möglichkeit zu vermeiden. Grundsätzlich sprechen sich die Empfehlungen für eine Erhöhung der Antihistaminika auf die bis zu vierfache normale therapeutische Dosierung aus. Dabei gilt es jedoch unbedingt zu beachten, dass die Verantwortung bei dem behandelnden Arzt liegt und eine ausführliche Beratung und Aufklärung des Patienten über mögliche Nebenwirkungen erfolgen muss. Obwohl hierfür ausschließlich nicht sedierende Antihistaminika verwendet werden sollten, muss insbesondere über eine Einschränkung der Vigilanz und des Reaktionsvermögens, z.B. im Straßenverkehr, informiert werden.

\section{PD Dr. Torsten Zuberbier}

Abteilung für Dermatologie und Allergologie, Charité-Virchow-Klinikum, Schumannstr. 20/21, D-10098 Berlin

\section{Kongress-Ankündigung: Garmisch-Partenkirchen, 1.-8. September 2001}

\section{Umwelt und genetische Einflüsse auf die menschliche Gesundheit \\ Mit folgenden Tagungen: \\ 2.-5. September 2001 (Eröffnung 1. September): 13. Jahrestagung der 'International Society for Environmental Epidemiology'(ISEE) \\ 2.-4. September 2001: \\ 10. Jahrestagung der 'International Genetic Epidemiology Society' (IGES) \\ 4.-5. September 2001: \\ US-European Workshop 'Gene Environ- ment Research at the Interface of Toxicology and Epidemiology'(US-EU) \\ 6.-8. September 2001: \\ 9. Jahrestagung der 'Gesellschaft für Hygiene und Umweltmedizin' (GHU) gemeinsam mit der 5. Jahrestagung der 'International Society of Environmental Medicine' (ISEM) \\ 6.-7. September 2001: 9. Jahrestagung der 'Deutschen Arbeitsge- meinschaft für Epidemiologie' (DAE)}

Registrierung und weitere Informationen unter http://www.gsf.de/epi/gap2001
Umwelt

- Feine und ultrafeine Partikel, kardiovaskuläre Effekte, Rolle des Verkehrs (ISEE 2.-5. Sept.)

- Prionenforschung in Mensch und Tier

(ISEE 3. Sept.)

- Elektromagnetische Felder (ISEE 4. Sept.)

- Klimatische Veränderungen (ISEE 4. Sept.)

Genetik

- Statistische Aspekte (IGES 2.-4. Sept.,

US-EU 5. Sept.)

- Gen-Umwelt-Interaktionen (IGES 3. Sept. US-EU 4.-5. Sept.)

- Gen-Umwelt-Forschung in USA und Europa, Toxikologie und Epidemiologie

(US-EU 4.-5. Sept.)

- Ethische Aspekte (US-EU 5. Sept.)

\section{Allergien}

- Verkehr und Allergien, Endotoxin und Allergien, frühe Einflüsse, Allergie in Deutschland (ISEE 4.-5. Sept.)

- Epidemiologie allergischer Erkrankungen (DAE 6. Sept.)

- Allergie und Umwelt, Hygiene und Allergien (GHU/ISEM 7. Sept.)

\section{Umweltmedizin}

- Multiple Chemical Sensitivity

(GHU/ISEM 7. Sept.)

- Klinische Umweltmedizin (GHU/ISEM 7. Sept.)

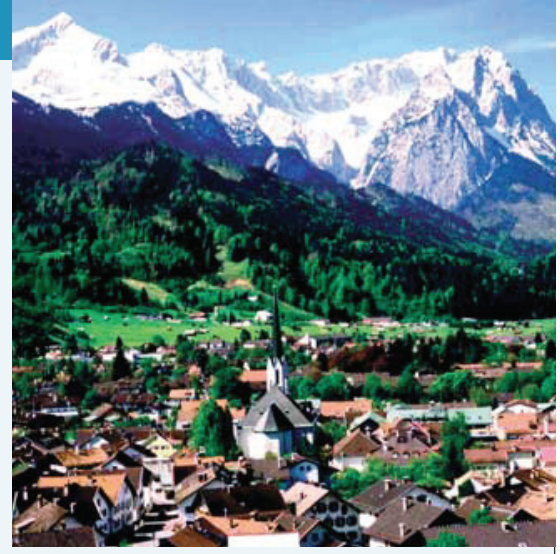

Konferenz Sekretariat

INTERPLAN

Albert-Rosshaupter-Strasse 65

D-81369 München

Tel +49-(0)89 / 5482 34-29

Fax +49-(0)89/ $548234-44$

E-mail: gap2001@i-plan.de

Gastgeber

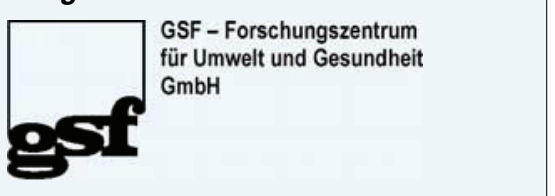

